\title{
The Therapeutic Efficacy of Domestic Violence Victim Interventions
}

\author{
Shannon Hackett', Paula T. McWhirter', and Susan Lesher'
}

\begin{abstract}
A meta-analysis on domestic violence interventions was conducted to determine overall effectiveness of mental health programs involving women and children in joint treatment. These interventions were further analyzed to determine whether outcomes are differentially affected based on the outcome measure employed. To date, no meta-analyses have been published on domestic violence victim intervention efficacy. The 17 investigations that met study criteria yielded findings indicating that domestic violence interventions have a large effect size $(d=.812)$, which decreases to a medium effect size when compared to control groups $(d=.518)$. Effect sizes were assessed to determine whether treatment differed according to the focus of the outcome measure employed: (a) external stress (behavioral problems, aggression, or alcohol use); (b) psychological adjustment (depression, anxiety, or happiness); (c) self-concept (self-esteem, perceived competence, or internal locus of control); (d) social adjustment (popularity, loneliness, or cooperativeness); (e) family relations (mother-child relations, affection, or quality of interaction); and (f) maltreatment events (reoccurrence of violence, return to partner). Results reveal that domestic violence interventions across all outcome categories yield effects in the medium to large range for both internalized and externalized symptomatology. Implications for greater awareness and support for domestic violence treatment and programming are discussed.
\end{abstract}

\section{Keywords}

domestic violence, battered women, children exposed to domestic violence, intervention/treatment.

The history of domestic violence intervention is characterized by salient changes in both scope and focus over time (Barner, 2011). One of the first catalysts for sweeping changes to domestic violence intervention was the "Battered Women's Movement" during the 1970's (Schechter, 1982). Schechter describes grassroots networks during this time period working to obtain government funding that would allow shelters to provide additional services to victims of intimate partner violence, rather than solely providing shelter and raising public awareness. Another factor that has contributed significantly to the changing face of domestic violence interventions developed in response to the growing understanding of the importance of safety for victims in the late 1970's. Stover (2005, p. 451) states that "assurance of physical and psychological safety for victims of domestic violence is a prerequisite for any other form of intervention. If women and children do not feel safe, it is impossible for them to engage in other forms of treatment." The availability and use of collaborative and community-based interventions have grown in an effort to address this fundamental need for safety. As a result of this ideological transition, a change was also seen as the focus of interventions shifted from victim centered to perpetrator centered. The Duluth Domestic Abuse Intervention Project (or the Duluth model) was the first interdisciplinary program designed to address IPV and was developed as a result of these ideological adjustments (Barner, 2011). Current intervention strategies tend to utilize group therapy approaches both for perpetrators (Austin \& Dankwort, 1999) and for victims (Danis, 2003).

\section{Intervention Effectiveness}

A wide range of techniques fall under the umbrella of domestic violence intervention. As a result, there is no clear consensus in the field about the efficacy of IPV intervention as a whole. Dutton (2012, p. 395) reviews many studies that yielded results ranging from "variable at best" to "notoriously unsuccessful." However, other studies offer support for the efficacy of domestic violence interventions (Blodgett, Behan, Erp, Harrington, \& Souers, 2008; Coker, Smith, Whitaker, Le, Crawford, \& Flerx, 2012; Tetterton \& Farnsworth, 2011). One of the most promising IPV interventions for both children and adults seems to be those that use a community-action approach (DePrince, Labus, Belknap, Buckingham, \& Gover, 2012; Graham-Bermann, Lynch, Banyard, DeVoe, \& Halabu, 2007). The study conducted by DePrince, Labus, Belknap, Buckingham, and Gover

\footnotetext{
I The University of Oklahoma, Norman, OK, USA

Corresponding Author:

Paula T. McWhirter, The University of Oklahoma, 820 Van Vleet Oval, Room 306, Norman, OK 73019, USA.

Email: paulamcwhirter@ou.edu.
} 
(2012), for example, indicated that the community-based outreach resulted in lower reported distress in victims of IPV. Similarly, the study by Graham-Bermann, Lynch, Banyard, DeVoe, and Halabu (2007) indicated that a different community-based intervention carried out with children exposed to IPV led to substantial reductions in both internalizing and externalizing behaviors.

Although there have been a significant number of metaanalytic studies involving domestic violence, none have reviewed the literature on victim intervention efficacy. The two primary areas of meta-analytic studies have investigated (1) the effects of exposure to domestic violence on kids (Evans, Davies, \& DiLillo, 2008; Kitzmann, Gaylord, Holt, \& Kenny, 2003; Wolfe, Crooks, Lee, McIntyre-Smith, \& Jaffe, 2003) and (2) batterer intervention efficacy (Babcock, Green, \& Robie, 2004; Feder \& Wilson, 2005; Olver, Stockdale, \& Wormith, 2011). The most relevant and comprehensive review of the effectiveness of interventions for IPV combined studies in a narrative synthesis, investigating four categories of intervention: perpetrator, victim, couple, and child (Stover, Meadows, \& Kaufman, 2009). Unfortunately, this review was not a meta-analysis, included a very small number of interventions per group (7 perpetrator, 5 victim, 5 couple, and 4 child), and excluded important information necessary to best understand the effectiveness of IPV interventions. This study, therefore, is an effort to provide more clarity regarding the effectiveness of intervention programs for intimate partner violence aimed at victims and child witnesses and to examine intervention effectiveness through meta-analysis.

\section{Method}

\section{Selection of Studies}

The following two techniques were employed to locate studies for this meta-analysis: (a) manual searches of the electronic library databases (e.g., PsycInfo) and (b) reference sections of review articles (e.g., Cohen, Mannarino, Murray, \& Igelman, 2006; Rivett, Howarth, \& Harold, 2006; Tolan, Gorman-Smith, \& Henry, 2006). The computerized searches involved articles that included any combination of the following terms: domestic violence, family violence, spousal abuse, battering, intimate partner violence, and child. Of the initial 3,608 studies, only those published in peer-reviewed journals were assessed for eligibility, leaving 2,294 studies. This was done to increase the methodological rigor of the studies included, ensuring that the studies had been scrutinized and the effect sizes pulled from these studies would be accurate. Of these 2,294 studies, each was assessed to determine whether it met the criteria needed for inclusion in the study. Only outcome studies assessing the effectiveness of mental health interventions that included women victims of domestic violence and their children were selected for inclusion. First, only studies that were about domestic violence mental health interventions were included, leaving 194 articles for analysis. Second, the studies had to include a sample of mothers and children who have experienced domestic violence, eliminating 111 more articles for a total of 83 studies. After collecting these studies, each was assessed for eligible effect size data. Studies that did not provide enough information to calculate an effect size were not included, eliminating 66 studies (31 were qualitative in nature, 24 were about aspects of interventions not related to outcome, the remaining 11 authors were contacted for access to their data but did not respond, elected not to share their data set, or had unusable data). This left a total of 17 studies that met the inclusion criteria for this study (see Table 1). Of these studies, 11 included data for both treatment and control groups.

\section{Coding of Samples}

Studies were coded by one of the researchers with previous meta-analytic experience. The coder pulled participant demographics and study outcome measure of participant functioning pre-to-post intervention (i.e., conduct, psychological adjustment, self-concept, social adjustment, and mother-child relations). Outcome measures of functioning were then divided into the grouping variables (i.e., depression, recurrence of family violence, and behavior problems) provided in the articles. This allowed for calculation of the overall effect size. After effect sizes were determined, the studies were then categorized into either internalizing or externalizing symptoms. Each sample was then coded into one of the six outcome categories based on the intended effect of each domestic violence intervention. Based on the division used by Amato and Keith (1991) and those that were used frequently in the literature, these categories were (a) external stress (behavioral problems, aggression, or alcohol use); (b) psychological adjustment (depression, anxiety, or happiness); (c) self-concept (self-esteem, perceived competence, or internal locus of control); (d) social adjustment (popularity, loneliness, or cooperativeness); (e) family relations (motherchild relations, affection, or quality of interaction); and (f) maltreatment events (reoccurrence of violence, return to partner). These categories reflect outcomes most frequently studied in relation to domestic violence.

\section{Calculation of Effect Sizes}

The effect sizes were obtained in several different ways, but all were calculated from the standardized mean difference effect size. Effect sizes already calculated by the authors (e.g., Cohen's $d$ coefficient) yielded $5.17 \%$ of cases. When this method was not available, effect sizes were calculated either from means and standard deviations, $t$ scores, or chi-squares (yielding $85.63 \%$ of the total number of samples) using a formula provided by Lipsey and Wilson (2001) to ensure that the effect size remained uniform across studies. When standard deviations were not provided, one was estimated from available data. In the cases where means and standard deviations were not available, $t$ values and chi-squares were used to calculate the effect size (Lipsey \& Wilson, 2001) and such procedures were used in $9.20 \%$ of cases. The following signs were affixed to effect sizes to reflect intervention effectiveness: (a) when no comparison group was used, a positive sign indicated 
Table I. Description of Studies.

\begin{tabular}{|c|c|c|c|c|c|}
\hline & $n$ & $\begin{array}{l}\text { Type of } \\
\text { Intervention }\end{array}$ & $\begin{array}{l}\text { Control } \\
\text { Group }\end{array}$ & Assignment & Outcome Measure \\
\hline Beeble, Bybee, Sullivan, and Adams (2009) & 160 & Advocacy & Yes & Random & $\begin{array}{l}\text { Psychological adjustment, self- } \\
\text { concept, social adjustment }\end{array}$ \\
\hline $\begin{array}{l}\text { Franzblau, Echevarria, Smith, and van } \\
\text { Cantfort (2008) }\end{array}$ & 40 & Empowerment & Yes & Random & Psychological adjustment \\
\hline Crusto et al. (2008) & 282 & Advocacy & No & $\mathrm{N} / \mathrm{A}$ & $\begin{array}{l}\text { External stress, psychological } \\
\text { adjustment }\end{array}$ \\
\hline Lieberman, van Horn, and Ippen (2005) & 75 & Parent-child & Yes & Random & $\begin{array}{l}\text { External Stress, Psychological } \\
\text { Adjustment }\end{array}$ \\
\hline McDonald, Jouriles, and Skopp (2006) & 33 & Parent-child & Yes & Random & $\begin{array}{l}\text { External stress, psychological } \\
\text { adjustment, social support, } \\
\text { family relations, } \\
\text { maltreatment events }\end{array}$ \\
\hline Pennell and Burford (2000) & 384 & Parent-child & Yes & Volunteer & Maltreatment events \\
\hline Smith and Landreth (2003) & 43 & $\begin{array}{l}\text { Play therapy, } \\
\text { Parent-child }\end{array}$ & Yes & Volunteer & $\begin{array}{l}\text { External stress, psychological } \\
\text { adjustment, self-concept, } \\
\text { family relations }\end{array}$ \\
\hline Sullivan, Egan, and Gooch (2004) & 125 & $\begin{array}{l}\text { Cognitive } \\
\text { behavioral }\end{array}$ & No & $\mathrm{N} / \mathrm{A}$ & $\begin{array}{l}\text { External stress, psychological } \\
\text { adjustment, self-concept, } \\
\text { social support, family } \\
\text { relations, }\end{array}$ \\
\hline Tyndall-Lind, Landreth, and Giordano (200I) & 60 & Play therapy & Yes & Volunteer & $\begin{array}{l}\text { External stress, psychological } \\
\text { adjustment, self-concept, }\end{array}$ \\
\hline Kot, Landreth, and Giordano (1998) & 40 & Play therapy & Yes & Volunteer & $\begin{array}{l}\text { External stress, self-concept, } \\
\text { social support, maltreatment } \\
\text { events }\end{array}$ \\
\hline McWhirter (2006) & 77 & $\begin{array}{l}\text { Cognitive } \\
\text { behavioral }\end{array}$ & Yes & Volunteer & Self-concept, social support \\
\hline McWhirter (20II) & 94 & $\begin{array}{l}\text { Cognitive } \\
\text { behavioral, } \\
\text { empowerment }\end{array}$ & No & Random & $\begin{array}{l}\text { Psychological adjustment, self- } \\
\text { concept, social support, } \\
\text { maltreatment events }\end{array}$ \\
\hline
\end{tabular}

Note. N/A = not applicable.

that the group experienced better functioning postintervention; (b) when studies compared treatment groups with control groups, a positive sign indicated that a treatment group experienced better functioning; and (c) a negative sign indicated that the control group experienced better functioning.

\section{Results}

All results are compared to Cohen's (2006) effect size statistics so that 0.20 or less is a small effect size, 0.50 is a medium effect ize, and 0.80 or greater is a large effect size. Of the 17 studies,
348 effect sizes were calculated. These effect sizes were then averaged per study so that only one effect size was utilized in the calculation of the overall effect size. Averaging was used over other methods to provide a more holistic view of adjustment. The variability across effect sizes did not exceed what would be expected based on sampling error, thus all of the effect sizes are estimating the same population, $\chi^{2}(347, N=$ $2,975)=111.79, p=.091$. Rosenthal's fail-safe $N$ calculation showed that 4,478 unpublished studies would be needed to make the population effect size nonsignificant. See Table 2 for the list of effect sizes by study. 
Table 2. Effect Size Statistics by Study.

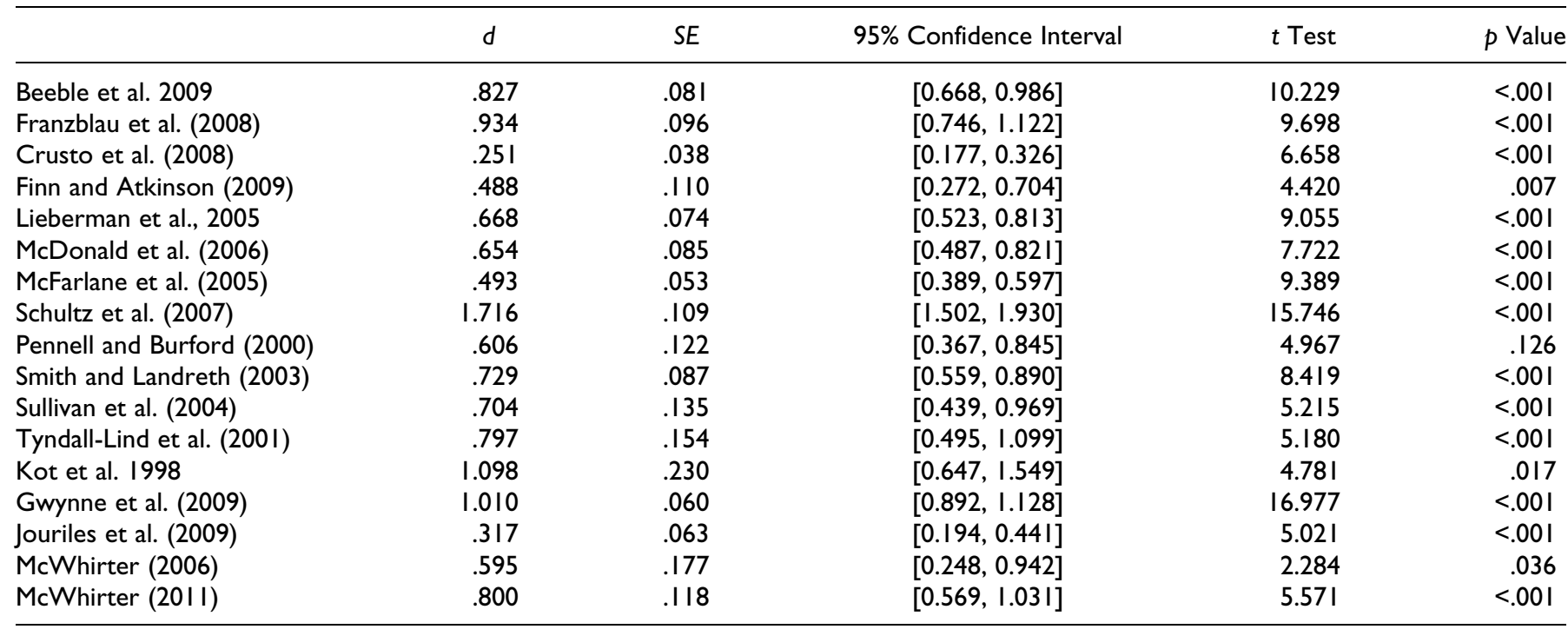

Note. $S E=$ standard error.

Table 3. Effect Size Statistics for Internalizing and Externalizing Symptoms.

\begin{tabular}{lcccr}
\hline & $d$ & $S E$ & $95 \%$ Confidence Interval & $t$ Test \\
\hline Internalizing & & & & \\
$\quad$ All & 0.749 & .054 & {$[0.643,0.950]$} & 13.209 \\
$\quad$ Without control & 0.846 & .065 & {$[0.653,0.97 I]$} & 12.026 \\
$\quad$ With control & 0.618 & .096 & {$[0.430,1.032]$} & 6.476 \\
Externalizing & & & {$[0.568,0.736]$} & $<.001$ \\
$\quad$ All & 0.652 & .043 & {$[0.680,0.884]$} & $<.001$ \\
Without control & 0.782 & .052 & {$[0.329,0.595]$} & $<.001$ \\
With control & 0.462 & .068 & & $<.001$ \\
\end{tabular}

Note. $S E=$ standard error.

The overall mean effect size was $d=0.755$ (standard error $[S E]=.088)$ which as shown by its $95 \%$ confidence interval $[0.583,0.928]$ and associated significance test $(t=8.599, p<$ $.001)$ differs significantly from zero, indicating that the domestic violence interventions have a medium-large effect on improving victim suffering.

Of the 348 effect sizes, 236 compared only the baseline with posttest for the intervention group. For those studies without a control group, the overall effect size was $d=0.812(S E=.032)$ which as shown by its $95 \%$ confidence interval [0.749, 0.875], and associated significance test $(t=21.979, p<.001)$ differs significantly from zero. According to the magnitude outline by Cohen, this is a "large effect size." This indicates that the intervention groups showed a significant improvement after they completed the intervention.

Next, the overall effect on adjustment was assessed for the 112 effect sizes that were compared to control groups. Mean differences from pre- to posttest were used on all comparison groups to ensure the highest amount of controllability. In many studies, the two groups had different scores at pretest so this eliminated any differences at the beginning to ensure that differences were due to treatment and not to pretest differences.
After aggregating these effect sizes as well, the overall effect size was $d=0.518(S E=.045)$ which as shown by its $95 \%$ confidence interval $[0.430,0.606]$, and associated significance test $(t=11.899, p<.001)$ differs significantly from zero. This indicates that there is a medium effect for interventions in comparison to control groups. Thus, interventions across studies do seem to have a significant effect on the well-being of the participants in comparison to those in the control group.

The outcome measures were then categorized into either internalizing or externalizing symptoms. The mean effect size for internalizing symptoms was $d=0.749$, indicating that the domestic violence interventions have a medium-large effect on these symptoms. Internalizing symptoms were then divided into whether or not they were compared to a control group. When studies without control groups were used, the effect size increased to $d=0.846$, indicating a still larger effect size. For the studies that utilized a control group, the effect size decreased to $d=0.618$, indicating that the internalizing symptoms improved among participants across studies, but to a lesser extent in studies involving comparison to control groups. The mean effect size for externalizing symptoms was $d=0.652$, indicating a medium effect size for interventions on externalizing 


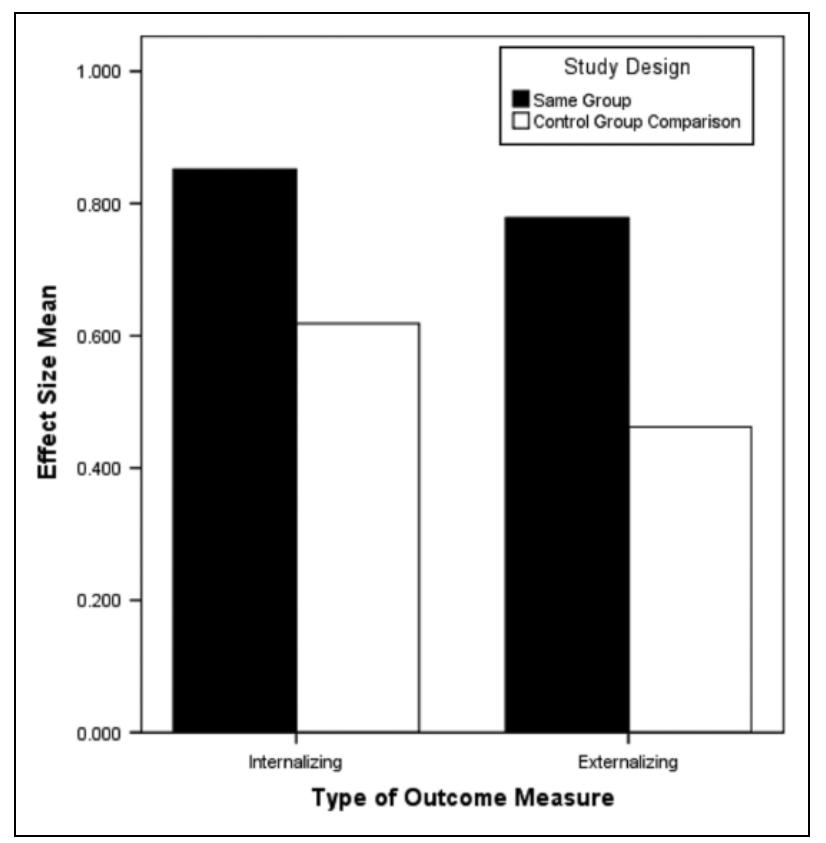

Figure I. Effect size means comparing outcome measure with study design.

symptoms. For studies that did not utilize control groups, the effect size increased to $d=0.782$, indicating that the effects of interventions on externalizing symptoms may be exaggerated without control group comparisons. Overall, the mean effect size decreased to $d=0.462$, indicating that intervention programs still have an impact on well-being (see Table 3 and Figure 1).

An independent samples $t$ test indicated that there were no significant differences between internalizing and externalizing symptoms for samples without control groups, $t(234)=0.663$, $p=.508$, nor with control groups, $t(90)=1.358, p=.178$, suggesting that both types of symptoms benefit equally from domestic violence interventions. Another independent sample $t$ test was run to determine whether having a control group significantly affects the resulting effect size. No significant difference was found for internalizing symptoms, $t(138)=1.413$, $p=.160$, indicating that using a control group does not significantly alter the effect size statistic. A significant difference was found, however, for externalizing symptoms, $t(186)=3.552$, $p<.001$, indicating that employing a control group results in a drop in effect size from large to medium.

The measures of adjustment were then assessed. The largest mean effect size was for maltreatment events, $d=1.118$, and the smallest was for family relations, $d=.478$, which indicated a medium-large amount of change related to intervention participation for all outcome measures (see Table 4 and Figure 2). The outcome measures were then divided into independent samples without control group comparisons or with control group comparisons. Without control groups, all effect sizes increased except self-concept which decreased slightly to $d=$ 0.720 . The largest mean effect size was still maltreatment events $(d=1.221)$ and the lowest was social adjustment $(d=0.697)$ indicating that all the effect sizes still fell in the medium-large effect of intervention (see Table 5). When only effect sizes with control groups were utilized, many of the outcome measures decreased to medium effect sizes. Self-concept, however, increased to $d=1.634$ and family relations decreased to $d=0.182$ (see Table 6). These results show that most outcomes are significantly impacted by domestic violence intervention programs.

Independent $t$ tests were run to determine whether there were any significant differences between groups that had a comparison group and those that did not. Significant differences were found for external stress, $t(128)=2.347, p=.020$; psychological adjustment, $t(100)=2.441, p=.016$; selfconcept, $t(39)=-3.764, p=.001$; and maltreatment events $t(11)=3.552, p=.005$. The difference between control group and no control group approached significance for social adjustment, $t(19)=1.942, p=.067$. The effect of intervention on family relations did not change with or without a control group, $t(19)$ $=0.860, p=.400$.

Finally, the six adjustment measures were divided between mothers and children. For mothers, all adjustment measures, except maltreatment events, were all medium effect sizes. Maltreatment events was a large effect size, $d=1.118$, indicating that domestic violence incidents significantly reduce at posttest for mothers. For child measures, all measures were shown to be medium-large effect sizes, except maltreatment events because this measure was only related to mothers in the studies (see Table 7).

Additional analyses were completed to determine whether significant differences existed between studies that utilized random assignment versus voluntary participation. These analyses revealed no significant differences. In particular, the effect size for studies with control groups with random assignment $(d=.640)$ and those with voluntary participation $(d=.731)$ were both medium effect sizes and not significantly different from one another, $t(244)=-1.342, p=.181$. Therefore, the full 17 studies were included in all analyses.

\section{Discussion}

The prevalence of domestic violence experienced by women and children is an alarming concern. If this trauma goes untreated, it can result in increased vulnerability to further experiences of victimization (McWhirter, 2006). In order to help victims and batterers recover, a variety of domestic and family violence intervention programs have been implemented. These interventions were developed for the batterers (usually men), adult victims (usually women), and the child victims of either direct or indirect violence. Over the past decade, intervention efficacy research has increased, providing both adult and child victim resources to help them move forward in the face of this devastating trauma.

Taken together, this meta-analysis reveals that domestic violence interventions have a significant and positive impact on the well-being of those who participate. Analysis of individual effect sizes was assessed to determine whether treatment differed according to the focus of the outcome measure employed: (a) external stress (behavioral problems, aggression, 
Table 4. Effect Size Statistics for Outcome Measures.

\begin{tabular}{|c|c|c|c|c|c|}
\hline & $d$ & SE & 95\% Confidence Interval & $t$ Test & $p$ Value \\
\hline Psychological adjustment & 0.757 & .064 & {$[0.632,0.882]$} & 10.837 & $<.001$ \\
\hline Social adjustment & 0.650 & .120 & {$[0.415,0.885]$} & 5.424 & $<.001$ \\
\hline Family relations & 0.478 & .138 & {$[0.208,0.749]$} & $3.47 I$ & .002 \\
\hline Maltreatment events & 1.118 & .096 & {$[0.930,1.306]$} & 11.697 & $<.001$ \\
\hline
\end{tabular}

Note. $S E=$ standard error.

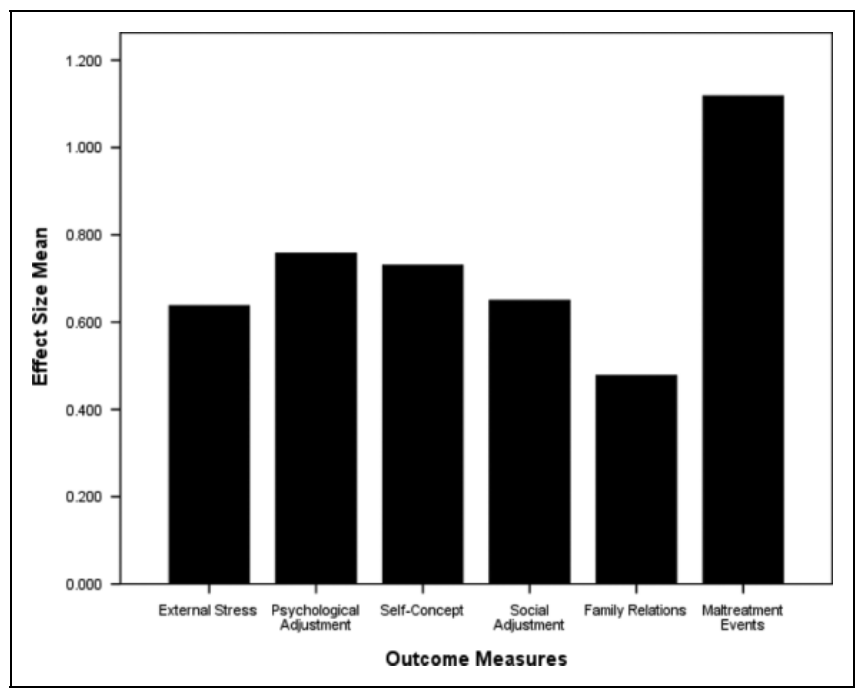

Figure 2. Effect size means across outcome measures.

or alcohol use); (b) psychological adjustment (depression, anxiety, or happiness); (c) self-concept (self-esteem, perceived competence, or internal locus of control); (d) social adjustment (popularity, loneliness, or cooperativeness); (e) family relations (mother-child relations, affection, or quality of interaction); and, (f) maltreatment events (reoccurrence of violence, return to partner). Results reveal that domestic violence interventions across all categories of outcome yield effects in the medium to large range. This finding is consistent with the majority of existing research. Most domestic violence intervention studies have found consistently positive and significant effects for (a) external stress (Graham-Bermann, Kulkarni, \& Kanukollu 2011; Grip, Almqvist, \& Broberg, 2012; McWhirter, 2011), (b) psychological adjustment (Crespo \& Arinero, 2010; DePrince et al., 2012; Teague, Hahna, \& McKinney, 2006), (d) social adjustment (Constantino, Kim, \& Crane, 2005; McNamara, Tamanini, \& Pelletier-Walker, 2008); and (e) family relations (Blodgett et al., 2008; Graham-Bermann, Lynch, Banyard, DeVoe, \& Halabu, 2007).

The studies investigating outcomes related to (c) self-concept are much less consistent than studies investigating the other outcomes mentioned earlier. The studies investigating global selfconcept (Brownell \& Heiser, 2006; Constantino et al., 2005; Teague et al., 2006) seem to yield inconclusive or nonsignificant results. However, when the treatment focuses on domain specific self-esteem such as safety self-esteem (Hughes et al., 2010) or career self-efficacy (Chronister \& McWhirter, 2006; Davidson, Nitzel, Duke, Baker, \& Bovaird, 2012), the results are much more consistently positive and significant.

Similar to (c) self-concept, the research related to (f) maltreatment events is also pretty inconsistent. Some studies (LaTaillade, Epstein, \& Werlinich, 2006; Todahl, Linville, Shamblin, \& Ball, 2012) indicate that domestic violence interventions are helpful in reducing future violent events. Other studies have yielded results that indicate positive, albeit nonsignificant effects (Coker et al., 2012; Stover, Berkman, Desai, \& Marans, 2010). Other research indicates that IPV interventions do not have an effect on reoccurrence of violence or return to the abusive partner (DePrince et al., 2012; Stover et al., 2009).

The fact that the effect size decreased when compared to control groups, however, lends support to the idea that time itself can affect adjustment; so controlling this effect with control groups is of critical importance. There have been a number of longitudinal studies that look specifically at the course of recovery from domestic violence without active intervention (Alsaker, Moen, \& Kristoffersen, 2008; Blasco-Ros, SanchezLorente, \& Martinez, 2010; Lindhorst \& Beadnell, 2011). Blasco-Ros, Sanchez-Lorente, and Martinez (2010) followed victims of IPV for 3 years and found that over this time period significant decreases occurred in depression, anxiety, and post-traumatic stress disorder symptoms. A comparison group of women who experienced purely psychological abuse (no physical abuse) was also followed, and it was found that this group did not experience the same reduction in symptomology. This difference was attributed to time after abuse because the physically abused women reported an $87.88 \%$ cessation of abuse, whereas the psychologically abused women only reported a $48.5 \%$ cessation of abuse. Another study conducted by Lindhorst and Beadnell (2011) also indicates that the passage of time affects adjustment following IPV. In this longitudinal study, teenage victims of IPV were followed for 17 years. It was found that different forms of IPV exposure predicted different psychosocial outcomes for roughly 13 years. However, by the time these women reached their 30 s, they had recovered to the point that there were no significant differences between groups. This again indicates that time can affect adjustment.

According to findings, outcome measures were not differentially affected by either parent or child measures, lending 
Table 5. Effect Size Statistics for Outcomes Measures Without a Control Group.

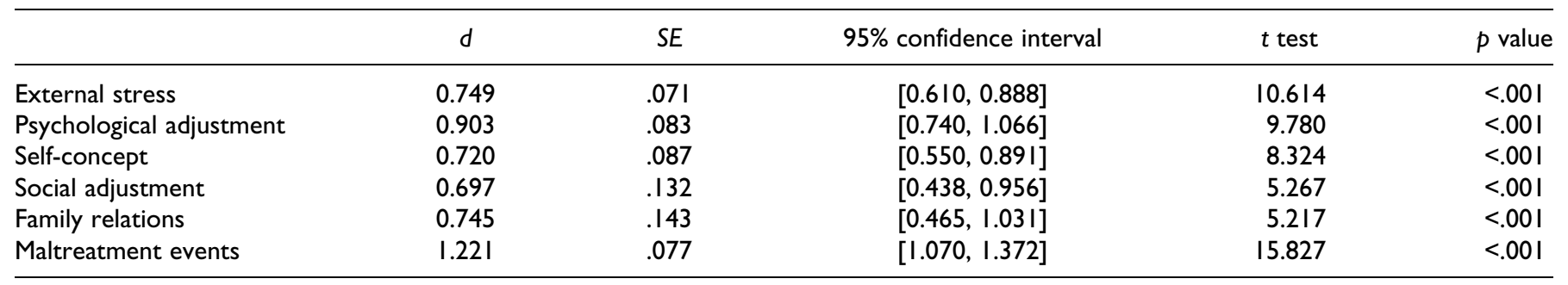

Note. $S E=$ standard error.

Table 6. Effect Size Statistics for Outcomes Measures With a Control Group.

\begin{tabular}{lccrrr}
\hline & $d$ & $S E$ & $95 \%$ Confidence Interval & $t$ Test & $p$ Value \\
\hline External stress & 0.498 & .073 & {$[0.355,0.641]$} & 6.838 \\
Psychological adjustment & 0.422 & .062 & {$[0.301,0.544]$} & 6.781 & $<.001$ \\
Self-concept & 1.634 & .182 & {$[1.277,1.991]$} & .001 \\
Social adjustment & 0.420 & .366 & {$[-0.297,1.137]$} & .991 & 1.149 \\
Family relations & 0.182 & .243 & {$[0.294,0.658]$} & .334 \\
Maltreatment events & 0.551 & .067 & {$[0.420,0.682]$} & .494 \\
\hline
\end{tabular}

Note. $S E=$ standard error.

Table 7. Effect Size Statistics for Outcome Measures Across Mothers and Children.

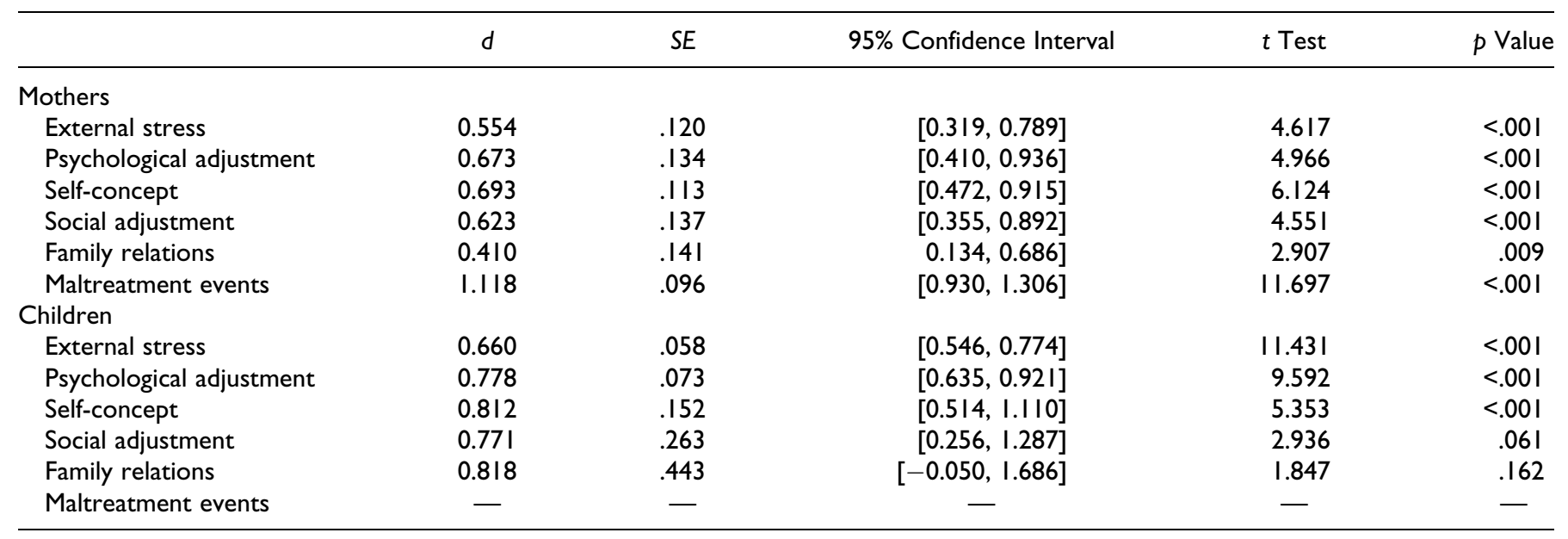

Note. $S E=$ standard error.

support to the idea that these interventions are effective at alleviating multiple types of maladjustment. This further supports the previous research that demonstrates mutually beneficial effects for both adults and children as a result of intimate partner violence interventions (Graham-Bermann et al., 2007; McWhirter, 2011). A review by Rizo, Macy, Ermentrout, and Johns (2011) demonstrates this further. This review examined the efficacy of IPV interventions with a child focus or child component and found a wide array of positive effects in both children and caregivers. One possible explanation for this observed tendency for interventions to alleviate multiple types of maladjustment can be found in a study conducted by Graham-Bermann, Howell, Lilly, and DeVoe (2011). In this study, the authors determined that better mental health (e.g., traumatic stress) on the part of the mother predicted a greater change in children's internalizing problems. This finding has profound implications for the design and implementation of future IPV intervention programs.

\section{Limitations}

We encountered a common limitation of meta-analytic research in that many published studies do not report all statistics needed to conduct the analysis; this is particularly true for "non-significant" findings. This is thought to result in findings that may inflate the perceived effectiveness of interventions studied. A total of 11 studies were not included in the current study due to a lack of appropriate data in the published article and an 
inability to receive the necessary data from the authors following attempts. These studies were not able to be included, and it is possible that this may have meaningfully impacted the current findings.

Further, the results could be biased due to an artifact of the publication process itself. Specifically, studies that demonstrate an intervention's lack of efficacy, or those that reveal an intervention as similar in outcome to its control, are generally less likely to be published. This also potentially skews data in the positive direction. Thus, it is impossible to know whether there are aspects of unpublished findings of domestic violence interventions that vary in efficacy, as these nonsignificant findings are often either unpublished or removed from published reports.

To compound these issues, intervention studies involving victims of domestic violence often do not utilize comparison groups. In many settings, acute need coupled with limited resources precludes use of a control group. Threats to internal validity, particularly history and maturation, make it difficult to contextualize intervention efficacy.

Finally, in terms of limitations, it should be noted that the current study was coded by one person, experienced in metaanalysis. Although the coding was subject to another's systematic random check for accuracy, it is possible that studies were missed for inclusion or that the included studies were miscoded.

\section{Conclusion}

The lack of public awareness about domestic violence interventions increases the likelihood that many experiencing domestic violence fail to seek the help that they need (Amnesty International Report, 2007). As this study suggests, current domestic violence intervention programs seem to effectively help victims cope with the trauma of their experiences. The authors hope that this meta-analysis may inform current practice, ultimately encouraging more effective outreach and treatment in domestic violence programming. Efforts should be made to publicize the availability of these programs and also for more agencies (domestic violence shelters, community agencies, etc.) to take the initiative to begin implementing the most effective programs. Further, increasing publicity of domestic violence intervention programs may play a role in instillation of hope, which may subsequently impact treatment selection, engagement, and outcome.

\section{Declaration of Conflicting Interests}

The author(s) declared no potential conflicts of interest with respect to the research, authorship, and/or publication of this article.

\section{Funding}

The author(s) received no financial support for the research, authorship, and/or publication of this article.

\section{References}

References marked with an asterisk indicate studies included in the meta-analysis.
Alsaker, K., Moen, B. E., \& Kristoffersen, K. (2008). Health-related quality of life among abused women one year after leaving a violent partner. Social Indicators Research, 86, 497-509.

Amato, P. R., \& Keith, B. (1991). Parental divorce and the well-being of children: A meta-analysis. Psychological Bulletin, 110, 26-46.

Amnesty International Report. (2007). Maze of injustice: The failure to protect indigenous women from sexual violence in the USA. New York, NY: Amnesty International USA.

Austin, J. B., \& Dankwort, J. (1999). Standards for batterer programs: A review and analysis. Journal of Interpersonal Violence, 14, $152-168$.

Babcock, J. C., Green, C. E., \& Robie, C. (2004). Does batterers' treatment work? A meta-analytic review of domestic violence treatment. Clinical Psychology Review, 23, 1023-1053.

Barner, J. R. (2011). Interventions for intimate partner violence: A historical review. Journal of Family Violence, 26, 235-244.

*Beeble, M. L., Bybee, D., Sullivan, C. M., \& Adams, A. E. (2009). Main, mediating, and moderating effects of social support on the well-being of survivors of intimate partner violence across 2 years. Journal of Consulting and Clinical Psychology, 77, 718-729.

Blasco-Ros, C., Sanchez-Lorente, S., \& Martinez, M. (2010). Recovery from depressive symptoms, state anxiety and post-traumatic stress disorder in women exposed to physical and psychological, but not to psychological intimate partner violence alone: A longitudinal study. BMC Psychiatry, 10, 98-109.

Blodgett, C., Behan, K., Erp, M., Harrington, R., \& Souers, K. (2008). Crisis Intervention for children and caregivers exposed to intimate partner violence. Best Practices in Mental Health, 4, 74-91.

Brownell, P., \& Heiser, D. (2006). Psycho-educational support groups for older women victims of family mistreatment. Journal of Gerontological Social Work, 46, 145-160.

Chronister, K. M., \& McWhirter, E. H. (2006). An experimental examination of two career interventions for battered women. Journal of Counseling Psychology, 53, 151-164.

Cohen, J. A., Mannarino, A. P., Murray, L. K., \& Igelman, R. (2006). Psychosocial interventions for maltreated and violence-exposed children. Journal of Social Issues, 62, 737-766.

Coker, A. L., Smith, P. H., Whitaker, D. J., Le, B., Crawford, T. N., \& Flerx, V. C. (2012). Effect of an in-clinic IPV advocate intervention to increase help seeking, reduce violence, and improve wellbeing. Violence Against Women, 18, 118-131.

Constantino, R., Kim, Y., \& Crane, P. A. (2005). Effects of a social support intervention on health outcomes in residents of a domestic violence shelter: A pilot study. Issues in Mental Health Nursing, 26, 575-590.

Crespo, M., \& Arinero, M. (2010). Assessment of the efficacy of a psychological treatment for women victims of violence by their intimate male partner. The Spanish Journal of Psychology, 13, 849-863.

*Crusto, C. A., Lowell, D. I., Paulicin, B., Reynolds, J., Feinn, R., Friedmann, S. R., \& Kaufman, J. S. (2008). Evaluation of a wraparound process for children exposed to family violence. Best Practices in Mental Health, 4, 1-18.

Danis, F. S. (2003). The criminalization of domestic violence: What social workers need to know. Social Work, 48, 237-245.

Davidson, M. M., Nitzel, C., Duke, A., Baker, C. M., \& Bovaird, J. A. (2012). Advancing career counseling and employment support for 
survivors: An intervention evaluation. Journal of Counseling Psychology, 59, 321-328.

DePrince, A. P., Labus, J., Belknap, J., Buckingham, S., \& Gover, A. (2012). The impact of community-based outreach on psychological distress and victim safety in women exposed to intimate partner abuse. Journal of Consulting and Clinical Psychology, 80, 211-221.

Dutton, D. G. (2012). The prevention of intimate partner violence. Prevention Science, 13, 395-397.

Evans, S. E., Davies, C., \& DiLillo, D. (2008). Exposure to domestic violence: A meta-analysis of child and adolescent outcomes. Aggression and Violent Behavior, 13, 131-140.

Feder, L., \& Wilson, D. B. (2005). A meta-analytic review of courtmandated batterer intervention programs: Can courts affect abusers' behavior? Journal of Experimental Criminology, 1, 223-233.

*Finn, J., \& Atkinson, T. (2009). Promoting the safe and strategic use of technology for victims of intimate partner violence: Evaluation of the technology safety project. Journal of Family Violence, 24, 53-59.

*Franzblau, S. H., Echevarria, S., Smith, M., \& van Cantfort, T. E. (2008). A preliminary investigation of the effects of giving testimony and learning yogic breathing techniques on battered women's feelings of depression. Journal of Interpersonal Violence, 23, 1800-1808.

Graham-Bermann, S. A., Howell, K. H., Lilly, M., \& DeVoe, E. (2011). Mediators and moderators of change adjustment following intervention for children exposed to intimate partner violence. Journal of Interpersonal Violence, 26, 1815-1833.

Graham-Bermann, S. A., Kulkarni, M., \& Kanukollu, S. (2011). Is disclosure therapeutic for children following exposure to traumatic violence? Journal of Interpersonal Violence, 26, 1056-1076.

Graham-Bermann, S. A., Lynch, S., Banyard, V., DeVoe, E. R., \& Halabu, H. (2007). Community-based intervention for children exposed to intimate partner violence: An efficacy trial. Journal of Consulting and Clinical Psychology, 75, 199-209.

Grip, K., Almqvist, K., \& Broberg, A. G. (2012). Maternal report on child outcome after a community-based program following intimate partner violence. Nordic Journal of Psychiatry, 66, 239-247.

*Gwynne, K., Blick, B. A., \& Duffy, G. M. (2009). Pilot evaluation of an early intervention programme for children at risk. Journal of Paediatrics and Child Health, 45, 118-124.

Hughes, R. B., Robinson-Whelen, S., Pepper, A. C., Gabrielli, J., Lund, E. M., Legerski, J., \& Schwartz, M. (2010). Development of a safety awareness group intervention for women with diverse disabilities: A pilot study. Rehabilitation Psychology, 55, 263-271.

*Jouriles, E. N., McDonald, R., Rosenfield, D., Stephens, N., CorbittShindler, D., \& Miller, P. C. (2009). Reducing conduct problems among children exposed to intimate partner violence: A randomized clinical trial examining effects of project support. Journal of Consulting and Clinical Psychology, 77, 705-717.

Kitzmann, K. M., Gaylord, N. K., Holt, A. R., \& Kenny, E. D. (2003). Child witnesses to domestic violence: A meta-analytic review. Journal of Consulting and Clinical Psychology, 71, 339-352.

*Kot, S., Landreth, G. L., \& Giordano, M. (1998). Intensive childcentered play therapy with child witnesses of domestic violence. International Journal of Play Therapy, 7, 17-36.
LaTaillade, J. J., Epstein, N. B., \& Werlinich, C. A. (2006). Conjoint treatment of intimate partner violence: A cognitive behavioral approach. Journal of Cognitive Psychotherapy: An International Quarterly, 20, 393-410.

*Lieberman, A. F., van Horn, P., \& Ippen, C. G. (2005). Toward evidence-based treatment: Child-parent psychotherapy with preschoolers exposed to marital violence. Journal of American Academy of Child and Adolescent Psychiatry, 44, 1241-1248.

Lindhorst, T., \& Beadnell, B. (2011). The long arc of recovery: Characterizing intimate partner violence and its psychosocial effects across 17 years. Violence Against Women, 17, 480-499.

Lipsey, M. W., \& Wilson, D. B. (2001). Practical meta-analysis. Thousand Oaks, CA: Sage Publications, Inc.

*McDonald, R., Jouriles, E. N., \& Skopp, N. A. (2006). Reducing conduct problems among children brough to women's shelters: Intervention effects 24 months following termination of services. Journal of Family Psychology, 20, 127-136.

*McFarlane, J. M., Groff, J. Y., O’Brien, J. A., \& Watson, K. (2005). Behaviors of children following a randomized controlled treatment program for their abused mothers. Issues in Comprehensive Pediatric Nursing, 28, 195-211.

McNamara, J. R., Tamanini, K., \& Pelletier-Walker, S. (2008). The impact of short-term counseling at a domestic violence shelter. Research on Social Work Practice, 18, 132-136.

*McWhirter, P. T. (2006). Community therapeutic intervention for women healing from trauma. The Journal for Specialists in Group Work, 31, 339-351.

*McWhirter, P. T. (2011). Differential therapeutic outcomes of community-based group interventions for women and children exposed to intimate partner violence. Journal of Interpersonal Violence, $X X, 1-27$.

Olver, M. E., Stockdale, K. C., \& Wormith, J. S. (2011). A metaanalysis of predictors of offender treatment attrition and its relationship to recidivism. Journal of Consulting and Clinical Psychology, 79, 6-21.

*Pennell, J., \& Burford, G. (2000). Family group decision making: Protecting children and women. Child Welfare, 79, 131-158.

Rivett, M., Howarth, E., \& Harold, G. (2006). 'Watching from the Stairs': Towards an evidence-based practice in work with child witnesses of domestic violence. Clinical Child Psychology and Psychiatry, 11, 103-125.

Rizo, C. F., Macy, R. J., Ermentrout, D. M., \& Johns, N. B. (2011). A review of family interventions for intimate partner violence with a child focus or child component. Aggression and Violent Behavior, 16, 144-166.

Schechter, S. (1982). Women and male violence: The visions and struggles of the battered women's movement. Boston, MA: South End Press.

*Schultz, P. N., Remick-Barlow, G. A., \& Robbins, L. (2007). Equine-assisted psychotherapy: A mental health promotion/ intervention modality for children who have experienced intrafamily violence. Health and Social Care in the Community, 15, 265-271.

*Smith, N., \& Landreth, G. (2003). Intensive filial therapy with child witnesses of domestic violence: A comparison with individual and 
sibling group play therapy. International Journal of Play Therapy, $12,67-88$.

Stover, C. A. (2005). Domestic violence research: What have we learned and where do we go from here? Journal of Interpersonal Violence, 20, 448-454.

Stover, C. S., Berkman, M., Desai, R., \& Marans, S. (2010). The efficacy of a police-advocacy intervention for victims of domestic violence: 12 month follow-up data. Violence Against Women, 16, 410-425.

Stover, C. S., Meadows, A. L., \& Kaufman, J. (2009). Interventions for intimate partner violence: Review and implications for evidence-based practice. Professional Psychology: Research and Practice, 40, 223-233.

*Sullivan, M., Egan, M., \& Gooch, M. (2004). Conjoint interventions for adults victims and children of domestic violence: A program evaluation. Research on Social Work Practice, 14, 163-170.

Teague, A. K., Hahna, N. D., \& McKinney, C. H. (2006). Group music therapy with women who have experienced intimate partner violence. Music Therapy Perspectives, 24, 80-86.

Tetterton, S., \& Farnsworht, E. (2011). Older women and intimate partner violence: Effective interventions. Journal of Interpersonal Violence, 26, 2929-2942.

Todahl, J., Linville, D., Tuttle Shamblin, A. F., \& Ball, D. (2012). Client narratives about experiences with a multicouple treatment program for intimate partner violence. Journal of Marital and Family Therapy, 38, 150-167.

Tolan, P., Gorman-Smith, D., \& Henry, D. (2006). Family violence. Annual Review of Psychology, 57, 557-583.
*Tyndall-Lind, A., Landreth, G. L., \& Giordano, M. A. (2001). Intensive group play therapy with child witnesses of domestic violence. International Journal of Play Therapy, 10, 53-83.

Wolfe, D. A., Crooks, C. V., Lee, V., McIntyre-Smith, A., \& Jaffe, P. G. (2003). The effects of children's exposure to domestic violence: A meta-analysis and critique. Clinical Child and Family Psychology Review, 6, 171-187.

\section{Author Biographies}

Shannon Hackett, MA, MEd, is in the final year of her $\mathrm{PhD}$ in Counseling Psychology at the University of Oklahoma. She has published four articles and conducted three meta-analyses on topics such as child adjustment to divorce, domestic violence victim interventions, and graduate student satisfaction. She is currently completing her internship at the University of California-Santa Barbara's Counseling and Psychological Services.

Paula T. McWhirter, PhD is Professor of Professional Counseling and Psychology in the Department of Educational Psychology at The University of Oklahoma. She researches the efficacy and effectiveness of counseling interventions for children, youth, and family across settings and cultures.

Susan Lesher is a Counseling Psychology Doctoral student at the University of Oklahoma, and is currently a doctoral intern of the Central Arkansas Veterans Healthcare System. She researches a variety of topics within the field of health psychology and is particularly interested in holistic program design, implementation and efficacy. 